\title{
Effects of glucose and yeast cream content on the Pelletization Behavior of Fungi-Chlorella Sp. Symbiosis System
}

\author{
Zou Yuchun ${ }^{1, a}$, Luo Shanshan ${ }^{1, b}$ and Li Wenkui ${ }^{2, c^{*}}$ \\ 1Department of Pharmacy, Jiangxi Science \& Technology Normal University, Nanchang, 330038, \\ CHINA \\ 2Key Lab of Surface Engineering, Jiangxi Science \& Technology Normal University, Nanchang, \\ 330038, CHINA \\ a18770053987, 'luoss82@163.com, 'liwenkui1976@163.com
}

\begin{abstract}
Keywords: Glucose, Yeast cream, Pelletization, Fungi, Chlorella sp..
Abstract. High cost of microalgae harvesting impeded the development of algae biofuel technology and wastewater treatment to commercial practicality. Filamentous fungi and microalgae could form pellets under certain culture condition and the symbiosis system is a novel method to reduce the cost of microalgae harvesting. In this paper, effects of glucose and yeast cream content on the pelletization behaviors of fungi and fungi-Chlorella sp. symbiosis system are investigated. The results show that the fungi are easier to form pellets with introduction of glucose or yeast cream.
\end{abstract}

\section{Introduction}

Due to the declining supplies of fossil energy resources and the contribution of these fuels to the accumulation of $\mathrm{CO}_{2}$ in the environment, much attention on the biomass-based biofuel production has been triggered[1]. Microalgae, with merits of environmental adaptability, high photosynthetic efficiency and high lipid content, have become one of the most promising raw materials for bio-fuels production. Since microalgae are also widely applied in waste water treatment, one promising way to make algae biofuel production more cost-effective is to couple wastewater treatment[2].

However, high cost of microalgae harvesting is a great challenge for algae biofuel technology and wastewater treatment to commercial practicality. The costs of harvesting process is generally accounting for $20-30 \%$ of the total costs of production[3]. Traditional algae harvesting methods, including centrifugation[4], filtration[5, 6] and flocculation[7], can only be applied in case of high value products accumulation in algae cells due to its high capital, energy and operational cost[8]. For ordinary microalgae culture solutions, such as Chlorella sp., low cell densities (typically $0.3-5 \mathrm{~g} / \mathrm{L}$ ) and small cell size (typically $1-30 \mu \mathrm{m}$ ) make the recovery of biomass very difficult. Some efforts, including using environmental flow diagram and computer modeling, has performed to reduce the costs of microalgae harvesting[9-11], however, the cost is still very high. Therefore, to reduce the energy consumption of harvesting microalgae, novel harvesting technology is needed.

Fungal have various morphology, including pellets, mycelia, clumps and the morphology could be controlled by adjusting culture parameters, including temperature, medium composition, viscosity, $\mathrm{pH}$, inoculums and additives etc[12]. There is great deal of valuable information concerning effect of these parameters on the pelletization behavior of fungi[13, 14]. Recently, Zhou and Zhang et al developed an effective fungal pelletization-assisted bioflocculation method for effective algae biomass harvesting and to apply the pelletized fungi -algae symbiosis system as immobilized cells to treat wastewater for improved nutrient removal and wastewater recycling[15-17]. Since the size 
of fungal pellets could reach more than several millimeters, the pelletized fungi -algae could be harvest feasibly by traditional filter screen.

The pelletization behavior of fungi-algae system is much more complicated than fungi alone, so further investigation on the effect of culture condition on that is needed. Glucose and yeast cream are commonly used as carbon and nitrogen resources in culture media. In this paper, effects of glucose and yeast cream content on the pelletization behavior of Fungal Chlorella sp. symbiosis system are investigated.

\section{Material and Methods}

\section{Algal and Fungal Strain Preparation}

Algal strain NCU C01 is isolated from local wastewater treatment plants and identified as Chlorella sp. The NCU C01 strain was able to grow on both the classic BG-11 medium under light and the modified BG-11 medium (in which 10g/L glucose was added) in the dark condition, suggesting that NCU 01 has both the autotrophic and the heterotrophic pathways and thus could be considered a facultative heterotrophic strain.

Aspergillus sp. NCU F01 is isolated from local environment and identified as Aspergillus sp. by morphological analysis. NCU F01 shows white color when grows on solid slant. The fungal species were stored in slant medium $(24 \mathrm{~g} / \mathrm{L}$ potato dextrose broth with $20 \mathrm{~g} / \mathrm{L}$ agar). The spore suspension was obtained by rinsing the slant with distilled water and the number of spores in the suspension was counted by using an optical microscope (B203LED, Chongqing Ao'te Optical and Scientific Instruments Inc., Chongqing, China).

\section{Experiment design:}

BG-11 medium, used in this study, contains following chemicals: $\mathrm{K}_{2} \mathrm{HPO}_{4} \cdot 3 \mathrm{H}_{2} \mathrm{O}(0.04 \mathrm{~g} / \mathrm{L})$, $\mathrm{MgSO}_{4} \cdot 7 \mathrm{H}_{2} \mathrm{O}(0.075 \mathrm{~g} / \mathrm{L}), \mathrm{CaCl}_{2} \cdot 2 \mathrm{H}_{2} \mathrm{O}(0.036 \mathrm{~g} / \mathrm{L})$, citric acid $(0.006 \mathrm{~g} / \mathrm{L})$, ferric ammonium citrate $(0.006 \mathrm{~g} / \mathrm{L})$, EDTA $(0.001 \mathrm{~g} / \mathrm{L}), \mathrm{NaNO}_{3}(1.5 \mathrm{~g} / \mathrm{L}), \mathrm{Na}_{2} \mathrm{CO}_{3}(0.02 \mathrm{~g} / \mathrm{L})$ and trace metal mix A5 (1.0ml). Trace metal mix A5 solution consisted of $\mathrm{H}_{3} \mathrm{BO}_{3}(2.86 \mathrm{~g} / \mathrm{L}), \mathrm{MnCl}_{2} \cdot 4 \mathrm{H}_{2} \mathrm{O}(1.81 \mathrm{~g} / \mathrm{L}), \mathrm{ZnSO}_{4} \cdot 7 \mathrm{H}_{2} \mathrm{O}$ $(0.222 \mathrm{~g} / \mathrm{L}), \mathrm{NaMoO}_{4} \cdot 2 \mathrm{H}_{2} \mathrm{O}(0.39 \mathrm{~g} / \mathrm{L}), \mathrm{CuSO}_{4} \cdot 5 \mathrm{H}_{2} \mathrm{O}(0.079 \mathrm{~g} / \mathrm{L})$ and $\mathrm{CoCl}_{2} \cdot 6 \mathrm{H}_{2} \mathrm{O}(0.05 \mathrm{~g} / \mathrm{L})$. pH was adjusted to 7.0 and $0-10 \mathrm{~g} / \mathrm{L}$ glucose, $0-9 \mathrm{~g} / \mathrm{L}$ yeast cream was added when needed.

The enriched Chlorella sp seed cultures were inoculated at $10 \%$ on $100 \mathrm{~mL}$ liquid medium in 250-mL Erlenmeyer flask, placed on a horizontal shaker $(150 \mathrm{rpm})$. The culture condition was kept at $26 \pm 2{ }^{\circ} \mathrm{C}$ with illumination by white incandescent lights $\left(100 \mu \mathrm{mol} / \mathrm{m}^{2} / \mathrm{s}\right)$. The initial inoculums sizes of fungal spores from $7 \mathrm{E} 4 / \mathrm{L}$ to $1.2 \mathrm{E} 9 / \mathrm{L}$ were added to algae culture medium for fungi -Chlorella sp. complex pellets formation.

\section{Results and Discussion}

As shown in Fig. 1, fungi pellets could not be formed without the introduction of glucose as while 0.5-2 mm pellets were formed after $72 \mathrm{~h}$ culture time with addition of glucose in range from 2 to $10 \mathrm{~g} / \mathrm{L}$. With glucose increasing from 2 to $6 \mathrm{~g} / \mathrm{L}$, more pellets formed, but no remarkable difference was observed when the glucose exceed $6 \mathrm{~g} / \mathrm{L}$. The results indicate that $\mathrm{C}$ resource is helpful for the formation of fungi pellets in present experiment condition. 

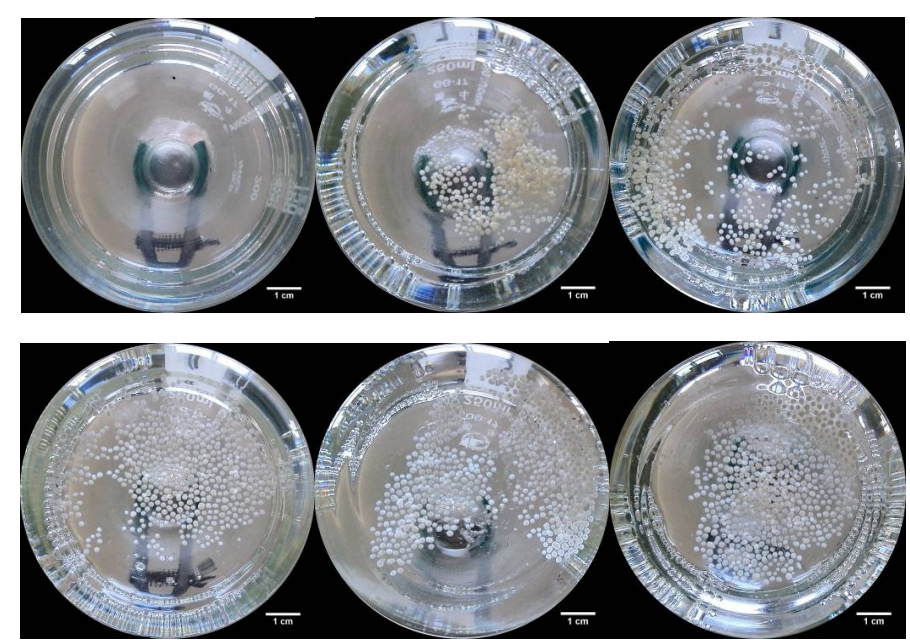

Fig. 1 Fungi pellets formed after $72 \mathrm{~h}$ cultivation (glucose $=0,2,4,6,8,10 \mathrm{~g} / \mathrm{L}$ )

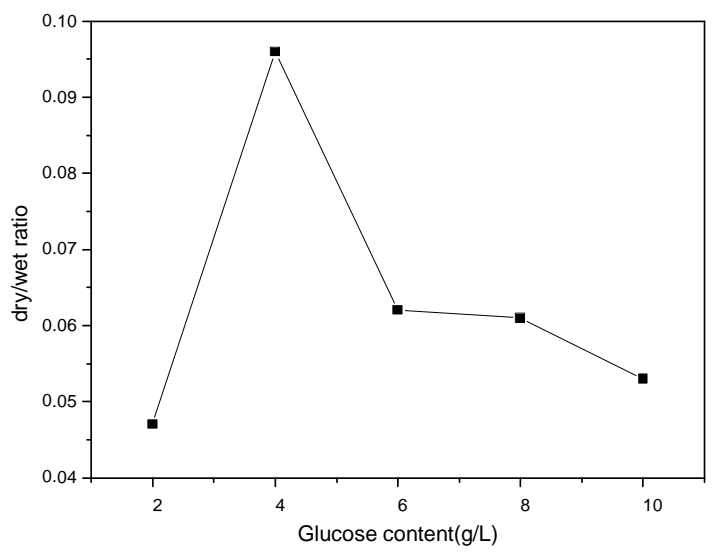

Fig. 2 Dry/wet weight ratios of pellets as function of glucose

Dry/wet weight ratios of pellets are shown in Fig. 2. When the glucose content is $4 \mathrm{~g} / \mathrm{L}$, pellets have the largest density.

In order to investigate the effect of yeast cream content on the pelletization behavior of fungi, yeast cream, ranging various from 1 to $9 \mathrm{~g} / \mathrm{L}$ were added into the culture media. Fig. 3 is the results of pelletization, where it can be seen that with increasing yeast cream, more pellets were formed. The pellets are looser larger than those of samples with only glucose added. Compared with the samples with only glucose, the samples with yeast cream tend to form clumps.

In the case of fungi and Chlorella sp. co-cultured with $2 \mathrm{~g} / \mathrm{L}$, and yeast cream ranged from 0 to $9 \mathrm{~g} / \mathrm{L}$, green pellets could be formed after $24 \mathrm{~h}$. After 72 culture period, the pellets turn into gray, which indicates the Chlorella sp. have died, as shown in Fig. 4. The size of pellets is similar to that in Fig. 3, which implies that the growth of Chlorella make little impact in fungi pellets. However, the Chlorella sp. inside the pellets has a shorter live period than others in the culture media. Possible reason is that the Chlorella sp. inside the pellets could not gain enough nutrient substances because the pellets impede the transport of nutrient from media to the centre of the pellets. 

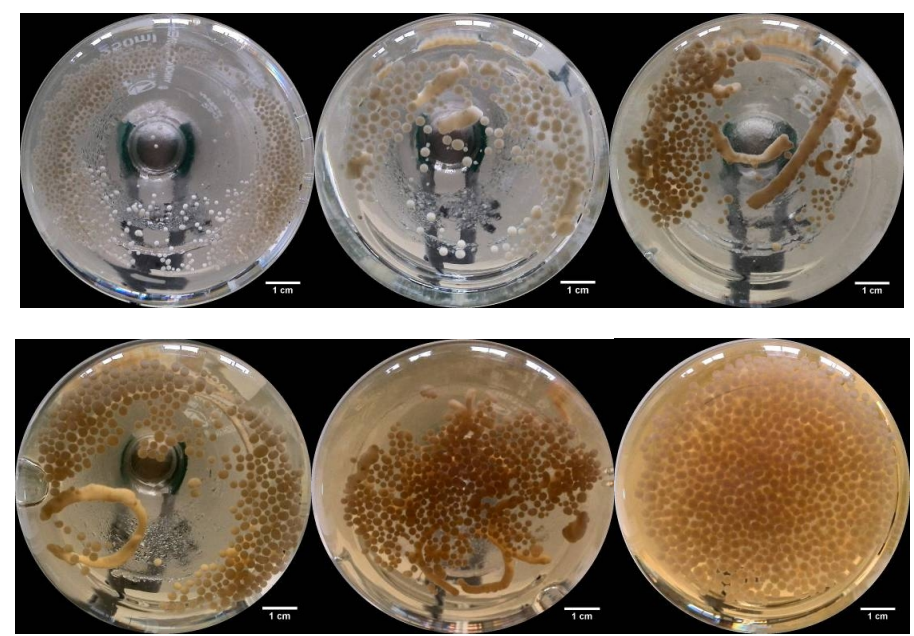

Fig. 3 Fungi pellets formed after $72 \mathrm{~h}$ with different yeast cream $($ glucose $=2 \mathrm{~g} / \mathrm{L}$, yeast cream $=0,1$, $3,5,7,9 \mathrm{~g} / \mathrm{L})$

The growth of Chlorella sp. will influence the $\mathrm{pH}$ value of the culture media. The start and final $\mathrm{pH}$ value of the culture media is shown in Fig. 5. Although the start $\mathrm{pH}$ value is decreased with increasing yeast cream content, the final $\mathrm{pH}$ value reaches 8.1-8.2 for all samples after $72 \mathrm{~h}$, which indicates that the Chlorella sp. has different grow rate with different yeast cream addition. The growth of Chlorella sp is sensitive to the $\mathrm{pH}$ value of the culture media. However more data need collected for thorough investigation and discussion on the relation between growth of fungi-Chlorella sp. pellets and the $\mathrm{pH}$ value.
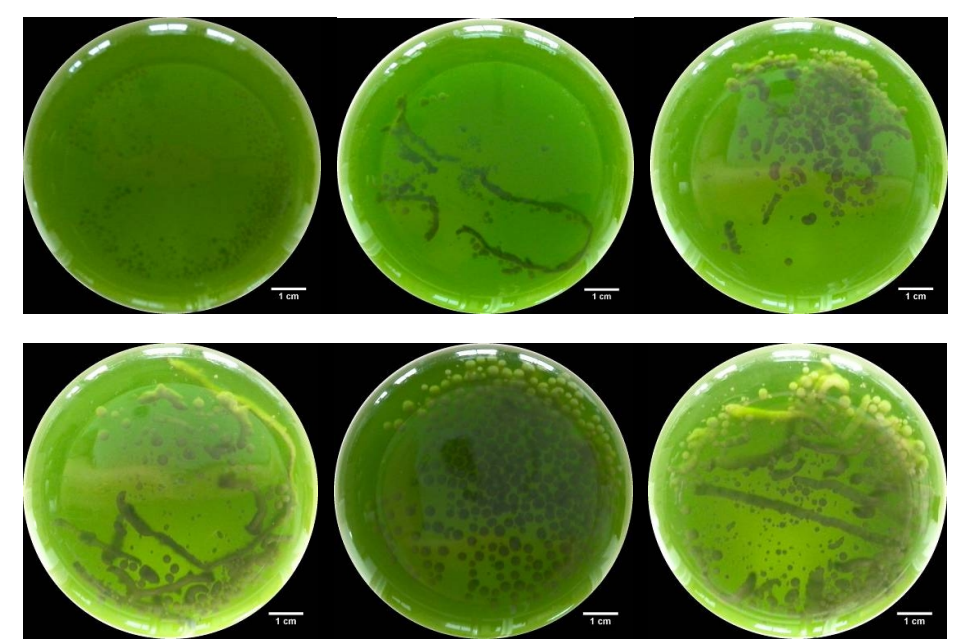

Fig. 4 Fungi-Chlorella sp pellets formed after $72 \mathrm{~h}$ culture. (Glucose $=2 \mathrm{~g} / \mathrm{L}$, yeast cream $=0,1,3,5,7,9 \mathrm{~g} / \mathrm{L})$ 


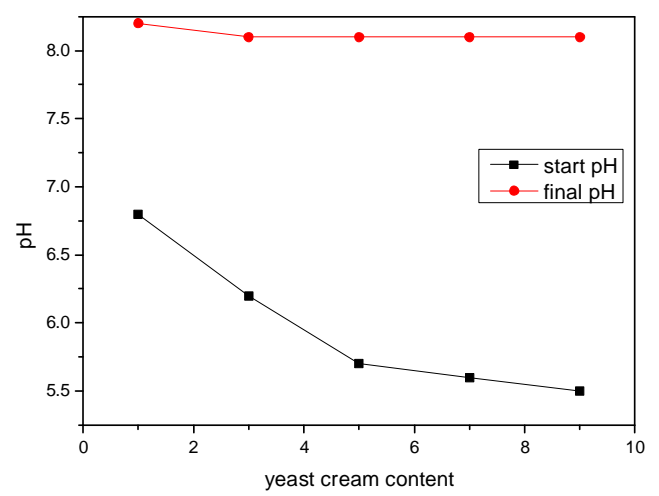

Fig. 5 Start and final $\mathrm{pH}$ value of fungi-Chlorella sp symbiosis system

\section{Conclusion}

Effects of glucose and yeast cream content on the pelletization behaviors of fungi Chlorella sp. are investigated. The results show that the introduction of either glucose or yeast cream is helpful to the formation of fungi pellets. The number of fungi pellets reach maximum at $6 \mathrm{~g} / \mathrm{L}$ glucose content. With increasing yeast cream, more fungi pellets could be formed. When fungi and Chlorella co-cultured, the growth of Chlorella sp make few impact on the pellets formation, but the pellets impede the transportation of nutrient form media to Chlorella sp inside pellets.

\section{Acknowledgement}

This work was supported by the National High-Tech R and D Program of China (2012AA101800) and Natural Science Foundation of China (21266022 ) .

\section{References}

[1] Y. Li, M. Horsman, N. Wu, C. Q. Lan, and N. Dubois-Calero, "Biofuels from microalgae," Biotechnology Progress, vol. 24, pp. 815-820, Jul-Aug 2008.

[2] L. Christenson and R. Sims, "Production and harvesting of microalgae for wastewater treatment, biofuels, and bioproducts," Biotechnology Advances, vol. 29, pp. 686-702, Nov-Dec 2011.

[3] J. J. Milledge and S. Heaven, "A review of the harvesting of micro-algae for biofuel production," Reviews in Environmental Science and Bio-Technology, vol. 12, pp. 165-178, Jun 2013.

[4] M. Heasman, J. Diemar, W. O'Connor, T. Sushames, and L. Foulkes, "Development of extended shelf-life microalgae concentrate diets harvested by centrifugation for bivalve molluscs - a summary," Aquaculture Research, vol. 31, pp. 637-659, Aug-Sep 2000.

[5] N. Rossi, O. Jaouen, P. Legentilhomme, and I. Petit, "Harvesting of cyanobacterium Arthrospira platensis usmg organic filtration membranes," Food and Bioproducts Processing, vol. 82, pp. 244-250, Sep 2004.

[6] X. Zhang, Q. Hu, M. Sommerfeld, E. Puruhito, and Y. Chen, "Harvesting algal biomass for biofuels using ultrafiltration membranes," Bioresource Technology, vol. 101, pp. 5297-5304, Jul 2010.

[7] E. Poelman, N. DePauw, and B. Jeurissen, "Potential of electrolytic flocculation for 
recovery of micro-algae," Resources Conservation and Recycling, vol. 19, pp. 1-10, Jan 1997.

[8] L. I. N. Zhe, K. Ya-li, G. U. O. Jin, and W. Zhang-guo, "A Review of Microalgae Recovery Technology," The Chinese Journal of Process Engineering, vol. 9, pp. 1242-1248, 2009 2009.

[9] M. Valipour, S. M. Mousavi, R. Valipour, and E. Rezaei, "Air, Water, and Soil Pollution Study in Industrial Units Using Environmental Flow Diagram," Journal of Basic and Applied Scientific Research and Essays, vol. 2, pp. 12365-12372, 2012.

[10] M. Valipour, S. M. Mousavi, R. Valipour, and E. Rezaei, "Deal with Environmental Challenges in Civil and Energy Engineering Projects Using a New Technology," Journal of Civil \& Environmental Engineering, vol. 3, p. 127, 2013.

[11] M. Valipour, S. M. Mousavi, R. Valipour, and E. Rezaei, "A New Approach for Environmental Crises and its Solutions by Computer Modeling," The 1st International Conference on Environmental Crises and its Solutions, Kish Island, Iran; 02/2013, 2013.

[12] R. Krull, T. Wucherpfennig, M. E. Esfandabadi, R. Walisko, G. Melzer, D. C. Hempel, et al., "Characterization and control of fungal morphology for improved production performance in biotechnology," Journal of Biotechnology, vol. 163, pp. 112-123, Jan 202013.

[13] J. Lee, D.-H. Cho, R. Ramanan, B.-H. Kim, H.-M. Oh, and H.-S. Kim, "Microalgae-associated bacteria play a key role in the flocculation of Chlorella vulgaris," Bioresource Technology, vol. 131, pp. 195-201, Mar 2013.

[14] S. O. Gultom and B. Hu, "Review of Microalgae Harvesting via Co-Pelletization with Filamentous Fungus," Energies, vol. 6, pp. 5921-5939, Nov 2013.

[15] J. G. Zhang and B. Hu, "A novel method to harvest microalgae via co-culture of filamentous fungi to form cell pellets," Bioresource Technology, vol. 114, pp. 529-535, Jun 2012.

[16] W. G. Zhou, Y. L. Cheng, Y. Li, Y. Q. Wan, Y. H. Liu, X. Y. Lin, et al., "Novel Fungal Pelletization-Assisted Technology for Algae Harvesting and Wastewater Treatment," Applied Biochemistry and Biotechnology, vol. 167, pp. 214-228, May 2012.

[17] W. Zhou, P. Chen, M. Min, X. Ma, J. Wang, R. Griffith, et al., "Environment-enhancing algal biofuel production using wastewaters," Renewable and Sustainable Energy Reviews, vol. 36, pp. 256-269, 2014. 